\title{
Influence of the Cholinergic Agonist RS 86 on Normal Sleep: Sex and Age Effects
}

\author{
Dieter Riemann, Daven Joy, Dominique Höchli, Christoph Lauer, Jürgen \\ Zulley, and Mathias Berger
}

Received November 17, 1986; revised version received May 29, 1987; accepted June 11, 1987.

\begin{abstract}
In 36 healthy control subjects (2l females, 15 males; age range 18-65 years; mean age 41.8 years, SD 15.6 years), a bedtime dose of $1.5 \mathrm{mg}$ RS 86 , an orally acting cholinergic agonist, shortened rapid eye movement (REM) latency, increased REM sleep, and decreased slow-wave sleep. Six of the subjects $(>40$ years old) even displayed sleep-onset REM periods after the drug. Results of the present study agree well with those of studies using other cholinomimetics (i.e., physostigmine, arecholine) and confirm the importance of the cholinergic system for REM sleep regulation. Since RS 86 mimicked some of the REM sleep abnormalities specific for patients with depressive disorders, the cholinergic system may play a role in the pathogenesis and pathophysiology of depressive diseases.
\end{abstract}

Key Words. RS 86, cholinergic system, rapid eye movement sleep, depression.

Much attention has focused on the "reciprocal interaction model of sleep regulation" (Hobson et al., 1975; McCarley and Hobson, 1975) as an explanation of the cyclic changes of non-rapid eye movement (NREM) and rapid eye movement (REM) periods during sleep. According to this theory, the regular patterning of NREM/REM sleep is governed by cell groups located in the brainstem. Ncurons in the locus ceruleus (via norepinephrinergic neurotransmission) are said to inhibit REM sleep, whereas neurons mainly in the pontine reticular formation (excited by acetylcholine and using it as a neurotransmitter) are believed to promote REM sleep. Reciprocal interaction between these transmitter systems is assumed to be responsible for the temporal structure of NREM/REM sleep. Empirical evidence for this theory is based on both animal and human studies. Direct injection of carbachol, an acetylcholine agonist, into the pontine reticular formation in cats reduced REM latency ( $=$ time from sleep onset to the occurrence of the first RFM period), increased REM period duration, and heightened amounts of REM sleep (for overview, see Hobson et al., 1986).

\footnotetext{
Dieter Riemann is Psychologist; Daven Joy is Research Assistant; Dominique Höchli, M.D., is Psychiatrist; Christoph l auer and .Jürgen Zulley, Ph.D., are Psychologists; and Mathias Berger, M.D., is Professor, Max-Planck-Institute of Psychiatry, Munich. D. Riemann, D. Joy, and Dr. M. Berger are now at the Central Institute of Mental Health, Mannheim. (Reprint requests to D. Riemann, Central Institute of Mental Health, Postfach 5970, 6800 Mannheim 1, F.R.G.)
}

0165-1781/88/\$03.50 1988 Elsevier Scientific Publishers Ireland Ltd. 
Such interaction in humans was tested with the cholinomimetic agents physostigmine and arecholine (for overview, see Sitaram et al., 1984). Intravenous administration of physostigmine or arecholine during the first or the second NREM period prompted an advance of the following REM period. These drugs are, however, accompanied by such practical limitations as a very short half-life, thus entailing protracted routes of administration like i.v. infusion during sleep. In addition, they have nonspecific effects on both central and peripheral muscarinic cholinergic receptors (physostigmine acts on nicotinic receptors as well), necessitating pretreatment with a peripheral muscarinic blocker like methylscopolamine.

Spiegel (1984) studied an alternative cholinergic agonist, RS 86. This orally acting muscarinic agonist has a half-life of about 8 hours (for pharmacology of RS 86, see Palacios et al., 1986) and minor side effects. RS $86,1.5 \mathrm{mg}$, administered p.o. to young healthy volunteers, disinhibited REM sleep during the first third of the night (i.e., shortened REM latency and increased REM sleep\%) and reduced slow-wave sleep (SWS) during the first two two-thirds of the night (Spiegel, 1984).

These results are of special interest for psychiatric research in depression, because the effects of RS 86 on sleep parameters in healthy controls mimic some of the sleep abnormalities characteristic of depression, such as shortened REM latency, prolonged duration and heightened density of the first REM period, and reduced SWS activity (for overview, see Gillin et al., 1984).

If these results are linked to the "cholinergic-adrenergic" hypothesis of mania and depression (Janowsky et al., 1972), which states that mania and depression are caused by a central nervous system (CNS) imbalance between the cholinergic and noradrenergic transmitter systems, REM sleep abnormalities in depression could be interpreted as indirect evidence of a pathophysiologically relevant dysregulation of neurotransmitters in depression (McCarley, 1982).

The aim of the present study was to replicate Spiegel's (1984) findings in a larger sample of healthy volunteers that included subjects of both sexes and older age groups and was thus more comparable to samples of depressed patients.

\section{Methods}

Design. RS $86,1.5 \mathrm{mg}$, was chosen as an appropriate dose to influence sleep (see Spiegel, 1984). Subjects slept in the laboratory for 3 nights, with night 1 serving for adaptation to the laboratory. Placebo or RS $86,1.5 \mathrm{mg}$, was administered in a double-blind, randomized design al $2200 \mathrm{~h}$, on nights 2 and 3.

Subjects. Thirty-six subjects (male $n=15$; female $n=21$ ) agreed to participate in the study and were paid for their services. Their mean age was 41.8 years (SD 15.6 years; range 18-65 years). Before being included in the study, subjects were thoroughly screened for physical and psychiatric disorders. They underwent a physical examination, electrocardiogram, drug screening, and an extensive psychiatric interview encompassing personal and family history of psychiatric disorders. Only subjects who were free of medical and psychiatric disorders and had no family history of affective disorders were included. Subjects received detailed information about the experimental procedures and possible side effects of the drug.

Mood Assessment. Before retiring and after waking up in the morning, subjects completed an adjective checklist, the AMS (von Zerssen, 1986) to gauge mood. 
Sleep. Sleep was recorded between lights out $(2315 \mathrm{~h})$ and lights on $(0630 \mathrm{~h})$ using standard procedures: horizontal electro-oculogram (EOG), submental electromyogram (EMG), and electroencephalogram (EEG) $\left(\mathrm{C}_{3}-\mathrm{A}_{2} ; \mathrm{C}_{4}-\mathrm{A}_{1}\right)$. Records were scored without knowledge of experimental condition by two experienced raters according to standardized criteria (Rechtschaffen and Kales, 1968).

The following sleep parameters were evaluated: (1) Sleep efficiency: ratio of total sleep time (TST) to time in bed (TIB) $\times 100 \%$. (2) Stages Wake, 1, 2, SWS (Stages 3 and 4 combined), and REM (expressed in \% of sleep period time (SPT), i.e., time from sleep onset until final awakening). (3) Latencies, i.e., time from beginning of the record to the first occurrence of Stage 2 (= sleep onset latency) and from sleep onset to the first occurrence of stage REM (= REM latency) (in min). (4) Number of awakenings during SPT. (5) Early morning awakening (EMA) (in min), i.e., time span between the last epoch of Stages 2, 3, 4, REM occurring during the record and before lights on in the morning. (6) Number of REM periods. (7) Duration of REM periods (in min). (8) NREM intervals: time span between REM periods (in min). (9) REM density for each REM period and for the whole night (REM density = the ratio of 3-sec mini-epochs per REM period including rapid eye movements to the total number of all 3-sec mini-epochs per REM period $\times 100 \%$ ).

Statistics. Descriptive statistical parameters include presentation of mean, standard deviation, and median. For comparisons between placebo and drug conditions, nonparametric methods were used: the Wilcoxon Test for dependent samples and the MannWhituey $U$ test for independent samples. The Spearmant rank-correlation coefficient served to assess correlations. To determine the differential effects of age and gender on sleep variables during placebo and drug conditions, a two-way analysis of variance (ANOVA) was conducted with the factors experimental condition (placebo vs. RS 86), gender (male vs. female), and age ( $\leqslant 40$ years vs. $>40$ years). For all calculations, the level of significance was set at $5 \%$ (two-tailed).

\section{Results}

Effects of RS 86 on Sleep. Table 1 displays results for the variables of sleep continuity, sleep architecture, and REM sleep for the whole sample, comparing placebo and drug condition. RS $86,1.5 \mathrm{mg}$, did not influence any parameter of sleep continuity. Regarding sleep architecture, the drug night was accompanied by a reduction in Stage 1 and SWS (\% SPT). REM sleep parameters were influenced in the expected manner. Mean REM latency was reduced by approximately $17 \min (p$ $<0.01)$ and Stage REM (\% SPT) was enhanced by about $4 \%(p<0.001)$. RS 86 had no influence on overall REM density and had no effect on the REM density of any of the first four REM periods. Fig. 1 illustrates the impact of $1.5 \mathrm{mg}$ RS 86 on the NREM/REM pattern until the end of REM period 4 (succeeding cycles were omitted due to decreasing case numbers).

As mentioned above, REM latency (expressed as $\mathrm{NREM}_{1}$ ) was shortened during the drug condition. The second NREM interval was also decreased $(p<0.001)$. REM period 1 was slightly but not significantly lengthened after RS 86 , while the length of REM period 2 was shortened in comparison to placebo $(p<0.05)$.

RS 86 significantly $(p<0.001)$ enhanced the mean ( \pm SD) number of REM periods in comparison to placebo (placebo: $3.8 \pm 0.8$; RS 86: 4.4 \pm 0.7 ).

Effects of RS 86 on Mood. Table 2 summarizes the results for mood assessment. As shown, RS 86 had no influence on parameters of well-being compared to placebo. 
Table 1. Influence of RS 86 on sleep in comparison with placebo in 36 healthy controls (mean \pm SD, median in parentheses)

\begin{tabular}{|c|c|c|c|}
\hline Sleep variables & Placebo & $\begin{array}{c}\text { RS } 86 \\
(1.5 \mathrm{mg})\end{array}$ & $\begin{array}{c}\text { Wilcoxon test } \\
\text { (2-tailed) }\end{array}$ \\
\hline \multicolumn{4}{|l|}{ Sleep continuity } \\
\hline Sleep efficiency (\%) & $\begin{array}{c}88.3 \pm 8.2 \\
(90.1)\end{array}$ & $\begin{array}{c}88.4 \pm 7.5 \\
(90.9)\end{array}$ & NS \\
\hline S-2 latency (min) & $\begin{array}{c}20.7 \pm 17.2 \\
\quad(14.8)\end{array}$ & $\begin{array}{c}19.4 \pm 12.0 \\
(17.0)\end{array}$ & NS \\
\hline Time awake ( $\%$ sleep period time) & $\begin{array}{c}6.0 \pm 6.0 \\
(3.5)\end{array}$ & $\begin{array}{c}6.2 \pm 5.4 \\
(5.2)\end{array}$ & NS \\
\hline No. wakenings & $\begin{array}{c}8.7 \pm 5.6 \\
(8.0)\end{array}$ & $\begin{array}{c}8.8 \pm 5.6 \\
(8.0)\end{array}$ & NS \\
\hline Early morning awakening (min) & $\begin{array}{c}5.2 \pm 13.2 \\
(0.5)\end{array}$ & $\begin{array}{c}4.7 \pm 10.2 \\
(0.5)\end{array}$ & NS \\
\hline \multicolumn{4}{|l|}{ Sleep architecture } \\
\hline Stage 1 (\% sleep period time) & $\begin{array}{c}8.5 \pm 5.2 \\
(6.7)\end{array}$ & $\begin{array}{c}7.5 \pm 4.2 \\
(6.3)\end{array}$ & $p<0.05$ \\
\hline Stage $2(\%$ sleep period time $)$ & $\begin{array}{c}55.4 \perp 7.6 \\
(54.3)\end{array}$ & $\begin{array}{c}56.0 \pm 9.3 \\
(55.1)\end{array}$ & NS \\
\hline SWS (\% sleep period time) & $\begin{array}{c}10.6 \pm 9.7 \\
(8.2)\end{array}$ & $\begin{array}{c}7.3 \pm 7.9 \\
(4.6)\end{array}$ & $p<0.001$ \\
\hline \multicolumn{4}{|l|}{ Rapid eye movement sleep } \\
\hline REM latency (min) & $\begin{array}{c}72.7 \pm 26.2 \\
(68.0)\end{array}$ & $\begin{array}{c}55.9 \pm 36.3 \\
(45.3)\end{array}$ & $p<0.01$ \\
\hline REM (\% sleep period time) & $\begin{array}{c}18.2 \pm 4.0 \\
\quad(18.2)\end{array}$ & $\begin{array}{c}21.9 \pm 5.8 \\
(22.4)\end{array}$ & $p<0.001$ \\
\hline REM density ( $\%$, whole night) & $\begin{array}{c}28.1 \pm 10.8 \\
(26.6)\end{array}$ & $\begin{array}{c}26.8 \pm 11.4 \\
(25.3)\end{array}$ & NS \\
\hline
\end{tabular}

Fig. 1. Effects of RS $86(1.5 \mathrm{mg})$ vs. placebo on the non-REM-REM cycle in 36 healthy controls

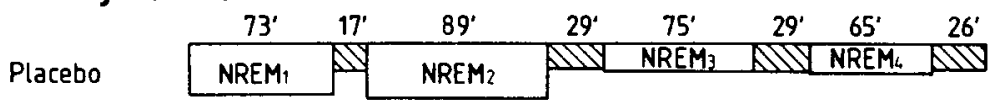

\begin{tabular}{|c|c|c|c|c|c|c|c|c|}
\hline & $56^{\prime * *}$ & $21^{\prime}$ & $69^{\prime * * *}$ & $22^{\prime *}$ & $69^{\prime}$ & $29^{\prime}$ & $65^{\circ}$ & $28^{\prime}$ \\
\hline $1.5 \mathrm{mg}$ RS 86 & NREM & NI & $\mathrm{NREM}_{2}$ & NII & $\mathrm{NREM}_{3}$ & DIII & NREM $_{L}$ & N1) \\
\hline
\end{tabular}

\begin{tabular}{llll}
\hline Sleep Onset $\quad 100$ & $200 \quad 300 \quad 400 \mathrm{~min}$. \\
& &
\end{tabular}

Note. REM = rapid eye movement. Length of open bars represents non-REM periods. Length of shaded bars represents REM periods. Bar width represents SD (same scale for $x$ - and $y$-axis). Asterisks indicate level of statistical significance (Wilcoxon test, 2-tailed) comparing placebo and RS 86: ${ }^{\star} p<0.05$; ${ }^{\star *} p<0.01$; $\star \star \star \rho<0.001$. 
Table 2. Influence of RS 86 on psychometric measurements in comparison with placebo in 36 healthy subjects (mean \pm SD, median in parentheses)

\begin{tabular}{lccc}
\hline & Placebo & $\begin{array}{c}\text { RS 86 } \\
(\mathbf{1 . 5} \mathbf{~ m g})\end{array}$ & $\begin{array}{c}\text { Wilcoxon test } \\
\text { (2-tailed) }\end{array}$ \\
\hline AMS evening & $17.9 \pm 8.2$ & $19.7 \pm 8.1$ & NS \\
AMS morning & $(19.0)$ & $(19.0)$ & NS \\
& $16.4 \pm 9.0$ & $16.9 \pm 9.3$ & \\
\hline
\end{tabular}

AMS $=$ adjective checklist to assess mood (von Zerssen, 1986).

Effects of Age and Gender. Fig. 2 illustrates the effect of RS 86 on REM latency when the sample was dichotomized by age: $\leqslant 40$ years ( 5 males, 8 females) vs. $>40$ years ( 10 males, 13 females). The mean ( \pm SD) REM latency in the younger age group decreased from $71.0 \pm 27.2 \mathrm{~min}$ to $61.1 \pm 30.7 \mathrm{~min}$ after $\operatorname{RS} 86(p<0.05)$, and this effect was even more pronounced in the older age group, with a decrease from $73.6 \pm 26.1 \mathrm{~min}$ to $52.8 \pm 39.4 \mathrm{~min}(p<0.01)$.

Six of the 23 older subjects ( 5 females), as compared with none of the younger subjects, showed a REM latency $<25$ min (SOREMP = sleep onset REM period) after RS 86. However, when REM latency for the whole sample was correlated with age for placebo, RS 86 , and the $\triangle \mathrm{REM}$ latency between the two conditions, the

Fig. 2. REM latencies for placebo and drug condition comparing young ( $\leq 40$ years) and old subjects $(>40$ years)
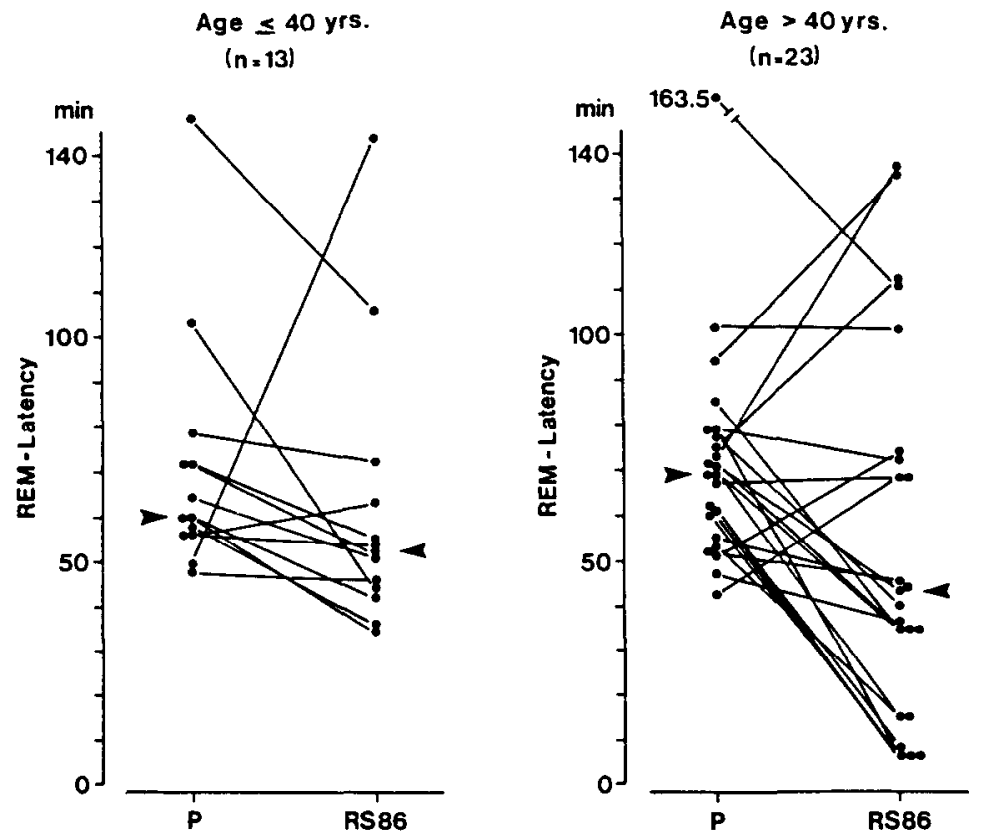

Note. $\mathrm{REM}=$ rapid eye movement. Arrows mark medians. 
following nonsignificant coefficients emerged: placebo, $r=-0.05 ; \operatorname{RS} 86, r=-0.26$; $\triangle$ REM latency, $r=-0.13$ (Spearman rank correlation). When $\Delta R E M$ latency (placebo - RS 86) was compared between the two age groups, no significant difference emerged ( $U$ test). A Fisher Exact Test comparing the occurrence of SOREMPs between the two age groups yielded an error probability of $p=0.10$. Upon closer scrutiny of Fig. 2, it does not seem surprising that no statistically verifiable higher responsiveness to the cholinergic stimulus occurred in the older subjects. As opposed to the six subjects with extremely shortened REM latency, five of the older volunteers displayed a moderate to marked delay of the first REM period after RS 86 in comparison to placebo. The same held true for 2 of the 13 younger subjects.

In an attempt to elucidate this paradoxical, unexpected lengthening of REM latency, the first NREM periods of all subjects were analyzed for the number of awakenings and Stages 1, 2, and SWS (min). Results from an earlier study (Berger et al., 1983) indicated that possible REM-inducing properties of cholinomimetics may be masked by sleep disruptions in some subjects due to the arousing properties of these drugs. Table 3 presents the number of awakenings and distribution of Stages Wake, 1, 2, and SWS separately for subjects with a shortened or lengthened REM latency after RS 86. Subjects with a shortened REM latency showed a decreased number of awakenings and duration of every sleep stage during NREM period 1 (statistically significant for Stage 2 and SWS). On the other hand, the seven subjects with a lengthened REM latency showed an increased number of awakenings, increased amounts of sleep Stages 1, and 2, and decreased SWS. The increase in Stages 1 and $2(\mathrm{~min})$ reached statistical significance, whercas there was only a strong tendency toward heightened Stage Wake after RS 86 during NREM period 1. The individual values of Wake time ( $\mathrm{min}$ ) after $\operatorname{RS} 86$ were $0,4,6,12,14.5,25.5$, and 32.5 min, indicating sleep disruption and heightened arousal in almost all of these subjects.

To evaluate age effects on all of the sleep variables, a two-way ANOVA was conducted with experimental condition (placebo vs. RS 86) and age ( $\leqslant 40$ years vs. $>40$ years) as factors. There were clear-cut age effects for many of the sleep variables. Sleep efficiency decreased, latency to Stage 2 increased, as did time awake (\% SPT) and the number of awakenings in the older age group. The older subjects spent more time in Stage 1 (\% SPT) and less time in SWS (\% SPT). REM density was diminished for the older subjects.

Effects due to experimental conditions were a reduction in SWS (\% SPT) and REM latency, and an enhancement of REM sleep (\% SPT). There were no significant interaction effects between age and experimental condition.

With regard to the effects of the experimental conditions and gender (male vs. female), no significant results were observed.

\section{Discussion}

Our results concerning the influence of my RS 86 on sleep in healthy subjects correspond well to those of other studies testing the effects of cholinomimetic agents on NREM/REM regulation. A dose of $1.5 \mathrm{mg}$ RS 86 caused a reduction of REM 


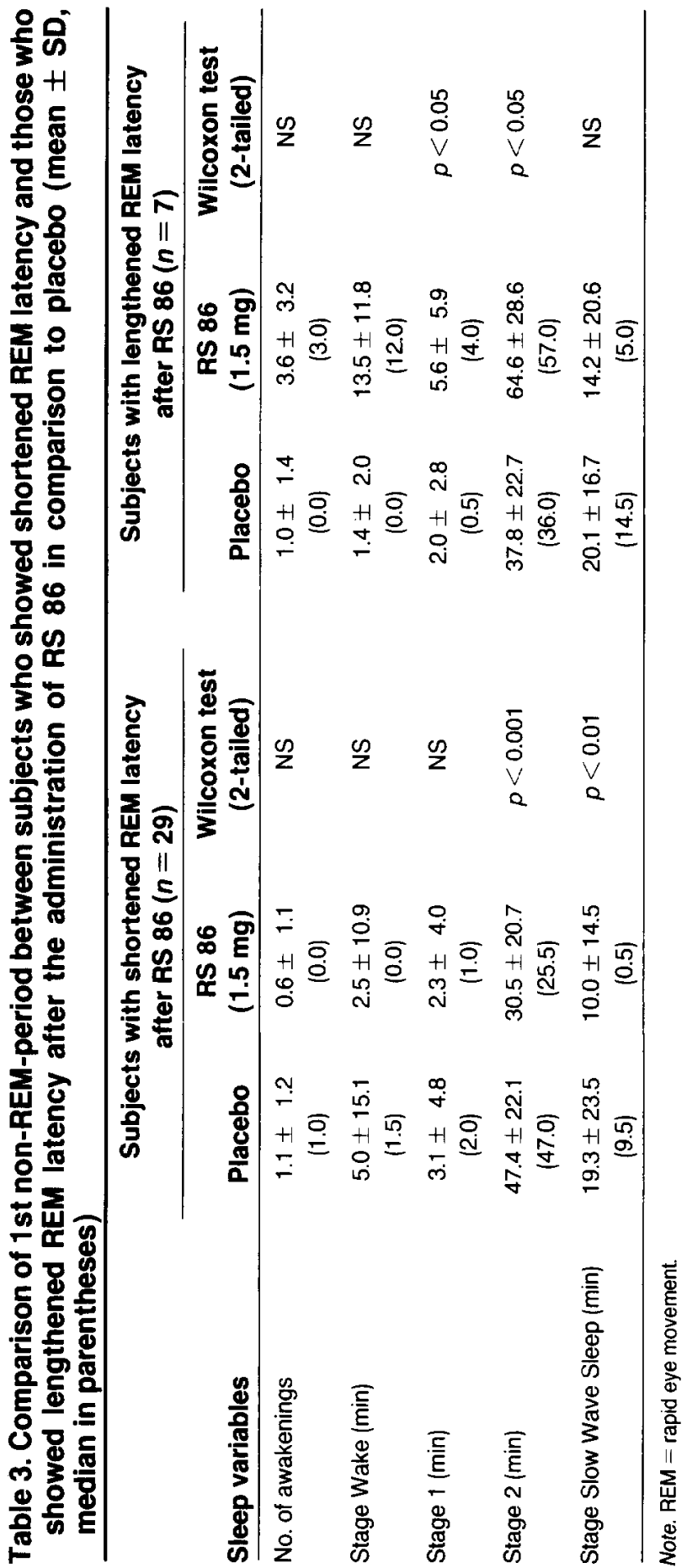


latency comparable to that provoked by physostigmine (Sitaram et al., 1976, 1977; Berger et al., 1983), arecholine (Sitaram et al., 1978a, 1978b), and RS 86 (Spiegel, 1984).

These results provide support for the hypothesis of a central cholinergic involvement in the regulation of REM sleep in humans. In comparison to the other cholinomimetic agents, which require more elaborate experimental designs, RS 86 offers a more convenient way of testing cholinergic involvement in the regulation of REM sleep. Its pharmacological properties (see Palacios et al., 1986) permit investigation of larger samples of control subjects and patients.

Though characterized by a long half-life, RS 86 exerted its effect on REM sleep mainly at the beginning of the night. RS 86 enhanced REM sleep (\% SPT) by $3.7 \%$ in comparison to placebo, though in view of the cyclic structure of NREM/REM patterns (see Fig. 1), it is evident that this effect is due to the shortening of the first (= REM latency) and second NREM periods, which led to a shift in the first REM-NREM cycle and thus to a heightened propensity for further REM periods to occur at the end of the night. In our study, RS 86 did not prolong the length of REM periods 1,3 , or 4, but it did induce a shortening of REM period 2, contrary to our expectation. This phenomenon is difficult to explain, especially in view of the long half-life of the drug. It may be speculated that counterbalancing reactions of those transmitter systems regulating the NREM/REM pattern neutralized the cholinergic impact. In accordance with all the other studies of cholinomimetics, RS 86 did not influence phasic aspects (i.e., eye movement density) of REM sleep. SWS (\% SPT) was significantly reduced by RS 86 . The decrease in SWS might be due to arousing properties of RS 86, an effect typical of all cholinergic agonists (Berger et al., 1983).

One explanation for shortened REM latencies, proposed by Borbély and WirzJustice (1982), stated that the reduction of SWS in depression (as measured by the power in the delta frequency band), due to a deficiency of process " $S$," may be the primary cause for REM sleep disinhibition at the beginning of the night. However, results of our studies (Berger et al., 1982, in press), as well as of studies of Van den Hoofdakker and Beersma (1985) do not support this hypothesis. Berger et al. (1982) found no correlation between REM latency and the amount of SWS in 45 depressive patients. In another study, Berger et al. (in press) showed that nine remitted depressives exhibited a highly significant reduction of SWS following $1.5 \mathrm{mg}$ RS 86, which was not accompanied by a significant shortening in REM latency. Van den Hoofdakker and Beersma (1985) also failed to find a relationship between REM latency and power in the delta frequency band (an even better estimate of SWS activity) in their sample of depressed patients. Therefore, it can be assumed that the shortening of REM latency observed in the present study was directly due to the REM-sleep-related properties of RS 86 . However, our results can provide only indirect evidence against the hypothesis of Borbély and Wirz-Justice (1982), since the SWS analysis was performed visually.

None of the sleep variables under investigation showed a significant gender-related effect. As for an age effect, baseline sleep data differed in the expected direction between the young and old subjects, confirming results reported in the literature (Williams et al., 1974; Feinberg, 1976). 
There were no significant interaction effects between age and drug condition. RS 86 caused SOREMPs in a subgroup of six (25\%) of the 23 older subjects. None of these subjects showed any psychopathological features. Absence of lifetime or family history of affective disorders had been established in prestudy interviews of the probands.

First-degree relatives were not interviewed directly - a necessary step, according to Gershon and Guroff (1984), in validating patients' or volunteers' statements about positive or negative family history. Therefore, it cannot be stated with finality that these subjects had no family history of affective disorders. Regarding the mood scores of these six subjects under RS 86 in comparison to placebo, no change was noted. Their placebo REM latencies were in the normal range (see Fig. 2), ruling out a preexisting elevation of their baseline level of cholinergic activity. Gillin et al. (1981) demonstrated that REM latency decreased as a function of age in both healthy subjects and depressed patients, being more pronounced in the latter group. They hypothesized that many of the sleep abnormalities found in depressive patients might be caused by a reversible process of premature aging in this group of patients. Our finding of a higher responsiveness of the REM sleep system to a cholinergic stimulus in a subgroup of older subjects could be interpreted as suggesting comparable changes in the neurotransmitter regulation of REM sleep during the physiological process of aging and in depression.

On the other hand, a paradoxical delay of REM latency after RS 86 was observed in seven of the subjects, five of whom belonged to the older group. A detailed analysis of their first NREM cycles suggested that in this group the administration of RS 86 led to an initial sleep disruption with more awakenings, Stage Wake, and Stage $I$ at the beginning of the night.

In summary, the effects of RS 86 on REM sleep in control subjects mimic some of the sleep abnormalities of depressive patients, thereby providing further indirect evidence of the cholinergic-aminergic hypothesis of REM sleep disinhibition and depression (Janowsky et al., 1972; McCarley, 1982).

In view of the facts that almost all antidepressant agents suppress REM sleep (for overview, see Chen, 1979), that the initial amount of REM sleep suppression during drug treatment may be a predictor of therapeutic outcome (Kupfer et al., 1983; Höchli et al., 1986), and that naps after successful sleep deprivation including REM sleep may lead to an immediate relapse (Wiegand et al., 1987), investigations of the neuronal and neurochemical processes responsible for the regulation of REM sleep are highly relevant. The cholinomimetic drug RS 86 seems to provide a useful tool for these investigations.

\section{References}

Berger, M., Doerr, P., Lund, R., Bronisch, T., and von Zerssen, D. Neuroendocrinological and neurophysiological studies in major depressive disorders: Are there biological markers for the endogenous subtype? Biological Psychiatry, 17, 1217 (1982).

Berger, M., Lund, R., Bronisch, T., and von Zerssen, D. REM latency in neurotic and endogenous depression and the cholinergic REM induction test. Psychiatry Research, 10, 113 (1983). 
Berger, M., Riemann, D., Höchli, D., and Spiegel, R. The cholinergic REM-sleepinduction test with RS 86: State or trait marker of depression. Archives of General Psychiatry, (in press).

Borbély, A.A., and Wirz-Justice, A. Sleep, sleep deprivation and depression. Human Neurobiology, 1, 205 (1982).

Chen, C.N. Sleep, depression and antidepressants. British Journal of Psychiatry, 135, 383 (1979).

Feinberg, J. Functional implications of changes in sleep physiology with age. In: Gershon, S., and Terry, R.D., eds. Neurobiology of Aging. Raven Press, New York (1976).

Gershon, E.S., and Guroff, J.J. Information from relatives: Diagnosis of affective disorders. Archives of General Psychiatry, 41, 173 (1984).

Gillin, J.C., Duncan, W.C., Murphy, D.L., Post, R.M., Wehr, T.A., Goodwin, F.K., Wyatt, R.J., and Bunney, W.E., Jr. Age-related changes in sleep in depressed and normal subjects. Psychiatry Research, 4, 73 (1981).

Gillin, J.C., Sitaram, N., Wehr, T.A., Duncan, W., Post, R.M., Murphy, D.L., Mendelson, W.B., Wyatt, R.J., and Bunney, W.E., Jr. Sleep and affective illness. In: Post, R.M., and Ballenger, J.C., eds. Neurobiology of Mood Disorders. Williams \& Wilkins Company, Baltimore (1984).

Hobson, J.A., Lydic, R., and Baghdoyan, H.A. Evolving concepts of sleep cycle generation: From brain centers to neuronal populations. Behavioral and Brain Sciences, 9, 371 (1986).

Hobson, J.A., McCarley, R.W., and Wyzinski, P.W. Sleep cycle oscillation: Reciprocal discharge by two brain stcm ncuronal groups. Science, 189, 55 (1975).

Höchli, D., Riemann, D., Zulley, J., and Berger, M. Initial REM sleep suppression by clomipramine: A prognostic tool for treatment response in patients with a major depressive disorder. Biological Psychiatry, 21, 1217 (1986).

Janowsky, D.S., El-Jousef, M.K., Sekerke, H.J., and Davis, J.M. A cholinergic-adrenergic hypothesis of mania and depression. Lancet, II, 632 (1972).

Kupfer, D.J., Spiker, D.G., Rossi, A., Coble, P.A., Ulrich, R., and Shaw, D. Recent diagnostic and treatment advances in REM sleep and depression. In: Clayton, P.J., and Barrett, J.E., eds. Treatment of Depression: Old Controversies and New Approaches. Raven Press, New York (1983).

McCarley, R.W. REM sleep and depression: Common neurobiological control mechanisms. American Journal of Psychiatry, 139, 565 (1982).

McCarley, R.W., and Hobson, J.A. Neuronal excitability modulation over the sleep cycle: A structural and mathematical model. Science, 189, 58 (1975).

Palacios, J.M., Bollinger, G., Closse, A., Enz, A., Gmelin, G., and Malanowski, J. The pharmacological assessment of RS 86 (2-ethyl-8-methyl-2, 8-diazaspero-4, 5-decan-1, 3-dionhydrobromide). A potent specific muscarinic acetylcholine receptor agonist. European Journal of Pharmacology, 125, 45 (1986).

Rechtschaffen, A., and Kales, A., eds. A Manual of Standardized Terminology, Techniques and Scoring System for Sleep Stages of Human Subjects. Department of Health, Education, and Welfare, Washington, DC (1968).

Sitaram, N., Gillin, J.C., and Bunney, W.E., Jr. Cholinergic and catecholaminergic receptor sensitivity in affective illness: Strategy and theory. In: Post, R.M., and Ballenger, J.C., eds. Neurobiology of Mood Disorders. Williams \& Wilkins Company, Baltimorc (1984). 
Sitaram, N., Mendelson, W.B., Wyatt, R.J., and Gillin, J.C. The time-dependent induction of REM sleep and arousal by physostigmine infusion during normal human sleep. Brain Research, 122, 562 (1977).

Sitaram, N., Moore, A.M., and Gillin, J.C. Experimental acceleration and slowing of REM sleep ultradian rhythm by cholinergic agonist and antagonist. Nature, 274, 490 (1978a).

Sitaram, N., Moore, A.M., and Gillin, J.C. Induction and resetting of REM sleep rhythm in normal man by arecholine: Blockade by scopolamine. Sleep, 1, 83 (1978b).

Sitaram, N., Wyatt, R.J., Dawson, S., and Gillin, J.C. REM sleep induction by physostigmine infusion during sleep. Science, 191, 1281 (1976).

Spiegel, R. Effects of RS 86, an orally active cholinergic agonist, on sleep in man. Psychiatry Research, 11, I (1984).

Van den Iloofdakker, R.H., and Beersma, D.G.M. On the explanation of short REM latencies in depression. Psychiatry Research, 16, 155 (1985).

Wiegand, M., Berger, M., Zulley, J., Lauer, Ch., and von Zerssen, D. The influence of daytime naps on the therapeutic effect of sleep deprivation. Biological Psychiatry, 22, 389 (1987).

Williams, H.L., Karacan, I., and Harsch, C.J. Electroencephalography (EEG) of Human Sleep. John Wiley \& Sons, New York (1974).

von Zerssen, D. Clinical Self-rating Scales (CSRS) of the Munich Psychiatry Information System (PSYCHIS München). In: Sartorius, N., and Ban, T., eds. Assessment of Depression. Springer, Berlin (1986). 